\title{
Bericht aus dem Arbeitskreis Psychiatrische Institutsambulanzen (AK PIA) - Gemeinsame Arbeitsgruppe der BDK und der ackpa
}

\section{Organisationsstruktur und inhaltlicher Auftrag}

Je Bundesland sind 1-2 Ambulanzleiter benannt, die mit den Sprechern zusammen den Arbeitskreis bilden. Sie planen, organisieren und bereiten die Jahrestagung vor (Kommunikations- und Informationsplattform für alle PIA-Verantwortlichen in Deutschland), die derzeit jeweils im Januar/Februar in Bielefeld stattfindet.

Der AK tagt am Vortag der Jahrestagung zur strategischen Entwicklung ambulanzpolitischer und -inhaltlicher Schwerpunkte. Weitere Treffen werden themenbezogen nach Bedarf vereinbart. Zuletzt stand das Thema intensiv-ambulante Behandlung (AMBI, s. u.) ganz im Mittelpunkt der Aktivitäten. Der AK verteilt regional und länderübergreifend kontinuierlich per EMail PIA-relevante Informationen und beantwortet Einzelfragen aus den PIAs. Übergeordnete Anfragen werden durch die AK-Leitung bearbeitet: bdk.pia@evkb. de.

PIA-bezogene Neuerungen und Diskussionen im AK-PIA aus dem letzten Berichtsjahr:

1. Psychosomatische Institutsambulanzen $(\S 118,3)$ - „Institutsambulanzen nach §118,3“ (offizielle Bezeichnung) Die Vereinbarungen wurden nach langen Verhandlungen im Herbst 2019 abgeschlossen. Die Umsetzung des Paragraphs wird wohl auf wenige Häuser beschränkt bleiben, da die Regelungen sehr eng sind. (Ermächtigung nur für Häuser ohne bereits bestehende PIA; Arbeit nur auf Überweisung durch Fachärzte aus dem Psych.Bereich; begrenzt auf definierte F-Diagnosen bei parallel bestehender somatischer Diagnose...)
2. „Omnibus-Regelungen“ im PT-Ausbildungs-Reformgesetz: Berufsgruppenübergreifende, koordinierte und strukturierte Versorgung

Im neuen Psychotherapeutengesetz wurde ein missverständlicher Halbsatz zum Problem. Für die Teilnahme an der vom GBA geplanten berufsgruppenübergreifenden koordinierten und strukturierten Versorgung schwer psychisch Kranker sollen Psychotherapeut*innen teilnehmen und dafür nach EBM vergütet werden, dies gelte auch für die Teilnahme der PIAs. Über die DGPPN wurde diese Problematik an die Politik herangetragen. Ergebnis: Sollte irgendeine Aktivität im Sinne der missverständlichen Interpretation erfolgen (z.B. Maßnahmen zur Veränderung der PIA-Vergütung in Richtung EBM seitens der GKV o. Ä.), werde eine gesetzliche Klarstellung dieser nicht intendierten Formulierung erfolgen.

Im PT-Reformgesetz wird eine weitere kritische Neuregelung festgeschrieben: Bei der Bestimmung der regionalen Bedarfsplanung soll künftig auch die Zahl der PIA-Ärzte einbezogen werden (Anrechnung von PIAs auf den regionalen Versorgungsgrad).

\section{Versorgung schwer psychisch Kranker} Derzeit (Sommer 2020) wird die Ausformulierung der berufsübergreifenden, koordinierten und strukturierten Versorgung in einer AG des GBA verhandelt, wichtig ist dabei aus Sicht des AK u. a. die Einordnung der PIAs in dieses ambulante Konzept. Im Herbst 2020 soll ein Entwurf veröffentlicht werden, zu dem die (auf Antrag zugelassenen) Verbände Stellung nehmen können. Für diesen Bereich der schwer psychisch Kranken ist die PIA als gesetzlich beauftragte Behandlungsinstitution zentral und hat sich inhaltlich über das Konzept „AMBI“ (s.u.) zusätzlich aufgestellt. Überraschenderweise stellte im Herbst 2019 die KBV-Vertragswerkstatt ein weit fortgeschrittenes Strukturkonzept aus dem vertragsärztlichen Sektor vor, das für die Gruppe komplex psychisch erkrankter Menschen die Ausweitung der ambulanten vertragsärztlichen Behandlung durch ein multiprofessionelles Team vorsieht. Vor dem Hintergrund der hohen Auslastung der Vertragsärzte sehen die PIA das Konzept kritisch.

\section{Ambulante Intensivbehandlung „AMBI“}

Bereits seit einigen Jahren ist es zentrales Thema des AK PIA, ob und wie eine intensiv-ambulante PIA-Behandlungsperspektive in die Regelversorgung implementiert werden kann. Diese Behandlung soll flexibel, ressourcenschonend, passgenau und so intensiv wie eben erforderlich sein und zwischen PIA-Regelbehandlung einerseits und StäB oder tagesklinischer Behandlung andererseits angesiedelt sein. Die Behandlung soll je nach Bedarf und Störungsbild sowohl innerhalb der PIA als auch aufsuchend stattfinden können. Das AMBI-Konzept erfüllt exakt die Forderungen des Sachverständigenrats-Gutachten vom Sommer 2018 und schließt die Lücke zwischen stationärem und regulär-ambulantem Behandlungsfeld.

Inzwischen liegt ein abgeschlossenes Konzeptpapier des AK-PIA vor, das von den Fachgesellschaften (DGPPN, BDK, ACKPA, LIPPS) konsentiert und auf den Homepages veröffentlicht wurde.

https://www.dgppn.de/presse/stellungnahmen/stellungnahmen-2020/AMBI. html

Das weitere Prozedere zur Umsetzung des AMBI-Modells wird nun zeitnah geplant.

gez. Dr. Steffi Koch-Stoecker und Prof. Dr. Martin Driessen, Klinik für Psychiatrie und Psychotherapie, Ev. Klinikum Bethel 